\title{
Design value of a headed stud shear resistance in composite steel - concrete beams - probability-based approach to evaluation
}

\author{
M. Maslak ${ }^{\mathrm{a} *}$ and T. Domanski ${ }^{\mathrm{a}}$ \\ ${ }^{a}$ Cracow University of Technology, Cracow, Poland \\ *corresponding author, e-mail address: mmaslak@pk.edu.pl
}

\begin{abstract}
Conventional standard procedure used to determine the design value of a headed stud shear resistance in composite steel-concrete beams is very simple but, in fact, mathematically incorrect, particularly in the case when such connector is automatically welded and when it is working in a solid slab. According to this approach the considered value is specified as a minimum of two separate design values. One of them is related to the resistance of the stud itself while the other is associated with the failure of the surrounding concrete. In the paper presented by the authors a new algorithm which allow to evaluate this value is recommended and discussed in detail. It seems to be more accurate because it is based on the fully probabilistic inference. In such approach a new random variable is introduced, being a minimum of two other, statistically independent, random variables. Analogously as it is in the concept previously mentioned, the first random variable quantifies now the steel stud shear resistance whereas the second one - the resistance of the adjacent concrete. Consequently, the sought design value is determined as a suitable quantile of this new random variable, characterized by log-normal probability distribution. It is shown that the design value of a headed stud shear resistance, calculated in this manner, strongly depends on the variability of strength parameters, relating both to the steel of which the connecting stud is made and to the concrete of the slab. In addition, it is found that in the case when the variability of concrete strength is too high, the safety factor recommended to use in European standards is not able to provide the required safety level, acceptable by the building users. The considerations presented in the article are illustrated by a detailed computational example.
\end{abstract}

Keywords: Headed stud; shear resistance; design value; probability analysis; log-normal probability distribution; strength variability.

\section{Introduction}

It is common knowledge that the headed studs are the conventional connectors widely used in composite steel - concrete structures to resist both horizontal shear and vertical uplift forces [1]. Much research has been carried out to estimate the strength of such studs. These were both the experimental tests and numerical simulations $[2,3]$. As a consequence, various equations have been proposed to specify the design value of a random shear resistance relating to the studs of this type. Among all the calculation procedures being available for use in this field the computational approach formalized in the standard EN 1994-1-1 [4, 5] is most often chosen by designers. According to this formal model, if the considered connector is placed in a concrete solid slab, the design value of a shear stud resistance $P_{R, d}$ is determined as the lesser of the design values $P_{R, s, d}$ and $P_{R, c, d}$. These values are given by two separate equations, one of which represents failure of the stud itself (a "steel failure") while the other - failure of the surrounding concrete. Consequently, there is:

$P_{R, d}=\min \left(P_{R, s, d}, P_{R, c, d}\right)$

where, in particular:

$$
P_{R, s, d}=0,8 f_{u} \frac{\pi d^{2}}{4} \frac{1}{\gamma_{v}}=\frac{P_{R, s, k}}{\gamma_{v}}
$$

and also: 


$$
P_{R, c, d}=0,29 \alpha d^{2} \sqrt{f_{c k} E_{c m}} \frac{1}{\gamma_{v}}=\frac{P_{R, c, k}}{\gamma_{v}}
$$

In the above mentioned formulae the quantities $P_{R, s, k}$ and $P_{R, c, k}$ are the characteristic values of the random resistances $P_{R, s}$ and $P_{R, c}$, respectively. This means that the coefficient $\gamma_{v}=1,25$ may be interpreted here as the partial safety factor specified specially for a considered connection. Furthermore, $f_{u}[M P a]$ - is the ultimate tensile strength of the steel from which the stud is made, $f_{c k}[M P a]$ - is the characteristic cylinder strength of the surrounding concrete, $E_{c m}[G P a]$ - is the secant modulus of elasticity of this concrete, $d[\mathrm{~mm}]$ is the diameter of the shank of a considered stud, $\alpha$ - is the coefficient taking into account the effective slenderness of such stud. In further analysis it is assumed that $h_{s c} / d>4$, where $h_{s c}[\mathrm{~mm}]$ is the stud length measured after welding. This allows to take that $\alpha=1,0$. The basic advantage of such a computational approach is simplicity, however, it is not fully correct in the mathematical sense. For this reason in this study the authors want to propose and to discuss in detail an alternative procedure, useful for more accurately determining the sought design value of a headed stud shear resistance. It is based on fully probabilistic calculations.

\section{Headed stud shear resistance as a new joint random variable}

\subsection{A single random implementation}

In our analysis both the resistance $P_{R, s}$ and the resistance $P_{R, c}$ are interpreted as the random variables. Moreover, it is assumed that these variables are statistically independent. Such a limitation seems to be justified because not only each of these quantities depends on different factors but also the failure modes corresponding to each of them can be analysed as formally separate cases. The small correlation between both random variables considered above, being a consequence of their common dependence on the stud diameter, is neglected in further considerations.

In order to determine for a single random implementation a representative value of a headed stud shear resistance $P_{R}$ it is necessary to choose a smaller value from a random pair of numbers $P_{R, s}$ and $P_{R, c}$. This means that:

$$
P_{R}=\min \left(P_{R, s}, P_{R, c}\right)
$$

Let $P_{R, s}=X$ and $P_{R, c}=Y$. In such an approach the random headed stud shear resistance can be defined by a new variable $Z$, such that:

$Z=\min (X, Y)$

It should be emphasized that the design value $Z_{d}$, specified for a joint random variable $Z$ which is defined as a minimum of two statistically independent random variables $P_{R, s}$ and $P_{R, c}$, is not equal to the minimum of design values $P_{R, s, d}$ and $P_{R, c, d}$ which were calculated separately for these variables [6].

\subsection{Cdf and pdf functions}

To identify a $c d f$ (cumulative distribution) function $F_{Z}(z)$, specified for a new random variable $Z$, it is necessary to integrate the joint $p d f$ (probability density) function, continuous by assumption and specified jointly for random variables $X$ and $Y$. The integration limits are in this case limited to the area in which the minimum $x$ and $y$ is smaller than $z$ [7]. This is also the complement of the area in which $x$ and $y$ are both greater than $z$ (Fig. 1). Hence:

$$
\begin{aligned}
& F_{Z}(z)=P(Z \leq z)=P[\min (X, Y) \leq z]= \\
& =1-P[\min (X, Y)>z]= \\
& =1-\int_{z}^{\infty} \int_{z}^{\infty} f_{X Y}(x, y) d x d y
\end{aligned}
$$

Thus, an appropriate $p d f$ function may be specified by a formula:

$$
\begin{aligned}
& f_{Z}(z)=\frac{d}{d z} F_{Z}(z)= \\
& =f_{X}(z)+f_{Y}(z)-f_{X}(z) F_{Y}(z)-f_{Y}(z) F_{X}(z)
\end{aligned}
$$

If the form of a continuous $p d f$ function $f_{Z}(z)$ is known in advance then two basic probabilistic moments of a random variable $Z$ may be calculated in a classic way. These are as follows:

- a mean value $\mu_{Z}=E(Z)$ as the first raw moment: 
$\mu_{Z}=\int_{-\infty}^{\infty} z f_{Z}(z) d z$

- a variance $\sigma_{Z}^{2}=\operatorname{var}(Z)$ as the second central moment:

$$
\sigma_{Z}^{2}=\int_{-\infty}^{\infty} f_{Z}(z)\left(z-\mu_{Z}^{2}\right) d z
$$

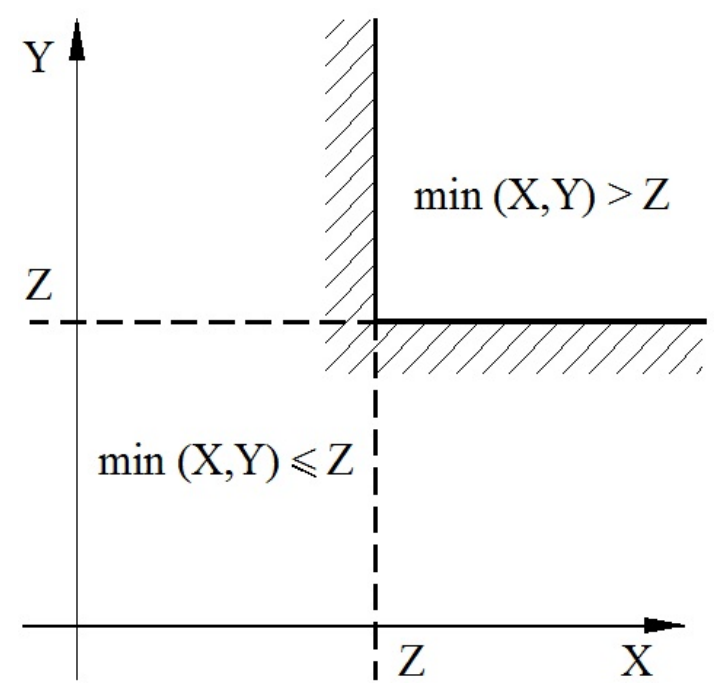

Fig. 1. The integration area used to determine the $c d f$ function specified for the random variable $Z$.

\subsection{Characteristics of a random variable $Z$ assuming its description by the log-normal probability distribution}

The random variable $Z$ has an interpretation of a headed stud shear resistance. For this reason the log-normal probability distribution is usually assigned to its description, because such distribution is specified only for $z \geq 0$ (i.e. in the range $0 \leq z<\infty)$. In this approach the random variable $\ln Z$ is characterised by the normal probability distribution described in the range $-\infty<\ln Z<\infty$. This means that:

$$
\begin{aligned}
& f_{Z}(z)= \\
& =\frac{1}{z \sigma_{\ln Z} \sqrt{2 \pi}} \exp \left\{-\frac{\left[\ln (z)-\mu_{\ln Z}\right]^{2}}{2 \sigma_{\ln Z}^{2}}\right\}
\end{aligned}
$$

Because the following occurs:

$\mu_{\operatorname{lnZ}}=\ln \breve{\mu}_{Z}$

where the quantity $\breve{\mu}_{Z}$ is a median value of the random variable $Z$, then:

$$
\breve{\mu}_{Z}=\exp \left(\mu_{\operatorname{lnZ}}\right)
$$

This value is quantitatively different than the analoguous mean value $\mu_{Z}=E(Z)$ calculated from the formula:

$$
\mu_{Z}=\exp \left(\mu_{\ln Z}+\frac{\sigma_{\ln Z}^{2}}{2}\right)
$$

Moreover, a variance $\sigma_{Z}^{2}=\operatorname{var}(Z)$ is equal to:

$$
\begin{aligned}
& \sigma_{Z}^{2}=\left[\exp \left(\sigma_{\ln Z}^{2}\right)-1\right] \mu_{z}^{2}= \\
& =\left[\exp \left(\sigma_{\ln Z}^{2}\right)-1\right] \cdot \exp \left(2 \mu_{\ln Z}+\sigma_{\ln Z}^{2}\right)
\end{aligned}
$$

which means that:

$$
\sigma_{\ln Z}=\sqrt{\ln \left(\frac{\sigma_{Z}^{2}}{\mu_{Z}^{2}}+1\right)}
$$

This allows to calculate the appropriate standard deviation:

$$
\sigma_{Z}=\sqrt{\operatorname{var}(Z)}=\mu_{Z} \sqrt{\exp \left(\sigma_{\ln Z}^{2}\right)-1}
$$

as well as the corresponding coefficient of variation:

$v_{Z}=\sqrt{\exp \left(\sigma_{\ln Z}^{2}\right)-1}$

\section{Specification of design and characteristic values for a random headed stud shear resistance}

A general safety condition defined for the log-normally standardized random variable $\ln (\breve{Z} / z)=\ln \left(\breve{\mu}_{Z} / z\right)$ has a form:

$$
\beta_{R}=\frac{\ln (\breve{Z} / z)}{v_{Z}} \geq \beta_{R, r e q}=\alpha_{R} \beta_{r e q}
$$

In this formula $\beta_{R}$ is a partial reliability index specified for a considered stud shear resistance while $\beta_{R \text {,req }}$ means the target value of such index setting the required safety level depending on the acceptable failure probability. Let us note that the failure is identified in our analysis as a random event in which the implemented value of a random headed stud shear resistance turns out to be smaller than the appropriate design value, determined earlier as a proper quantile of the log-normal probability distribution. Obviously, index $\beta_{R, \text { req }}$ is here only a part of a conventional global reliability index $\beta_{\text {req }}$ commonly used to verify the global safety condition type $E_{d} \leq Z_{d}=P_{R, d}$ (symbol $E_{d}$ denotes in this case the design value of an authoritative, most unfavourable, action effect of a combination of the loads applied to the considered stud). According to the standard EN 
1990 [8], for the ordinary safety requirements it is assumed that $\beta_{\text {req }}=3,8$, which is associated with the acceptable failure probability set at the level $p_{f, u l t} \approx 7,2 \cdot 10^{-5}$. Moreover, using the computational format recommended in [8] a fixed value $\alpha_{R}=0,8$ can be assumed in the analysis. This leads to the specification that $\beta_{R, r e q}=\alpha_{R} \beta_{\text {req }}=0,8 \cdot 3,8=3,04$.

Condition (18) is equivalent to the formula:

$P\left(Z<Z_{d}\right) \leq p_{f, u l t}=$

$=\Phi\left(-\beta_{R, r e q}\right)=\Phi\left(-\alpha_{R} \beta_{r e q}\right)$

Symbol $\Phi()$ means here the $c d f$ function of a standardized normal probability distribution. In other words, it is a well-known Laplace function with values compiled in the conventional statistical tables. If it is accepted that $\beta_{R, r e q}=3,04$ then, based on (19), the following occurs $p_{f, u l t} \approx 1,18 \cdot 10^{-3}$.

In a situation when the ultimate limit state (18) is reached the equality $Z=Z_{d}$ occurs. This allows to transform this formula to a form:

$\frac{\ln \left(\breve{Z} / z_{d}\right)}{v_{Z}}=\beta_{R, r e q}=\alpha_{R} \beta_{\text {req }}$

which gives:

$$
Z_{d}=\breve{Z} \exp \left(-\alpha_{R} \beta_{r e q} v_{Z}\right)=\breve{Z} \exp \left(-3,04 v_{Z}\right)
$$

By converting the equations (12) and (13) one can obtain that:

$$
\begin{aligned}
\breve{Z} & =\breve{\mu}_{Z}=\exp \left[\ln \mu_{Z}-\frac{\sigma_{\operatorname{lnZ}}^{2}}{2}\right]= \\
\frac{\mu_{Z}}{\exp \left(\frac{\sigma_{\ln \mathrm{Z}}^{2}}{2}\right)} & =\frac{E(Z)}{\exp \left(\frac{\sigma_{\ln \mathrm{Z}}^{2}}{2}\right)}
\end{aligned}
$$

This allows to describe a formula (21) in an alternative way:

$$
\begin{aligned}
& Z_{d}=\mu_{Z} \exp \left(-\alpha_{R} \beta_{r e q} v_{Z}-\frac{\sigma_{\ln Z}^{2}}{2}\right)= \\
& =E(Z) \exp \left(-3,04 v_{Z}-\frac{\sigma_{\ln Z}^{2}}{2}\right)
\end{aligned}
$$

The characteristic value of a random headed stud shear resistance is recommended here to be determined in a classic way, as a $95 \%$ quantile of the log-normal $p d f$ function $f_{Z}(z)$. This leads to a formula:

$Z_{k}=\breve{Z} \exp \left(-1,645 v_{Z}\right)=$

$E(Z) \exp \left(-1,645 v_{Z}-\frac{\sigma_{\operatorname{lnz}}^{2}}{2}\right)$

Based on the values $Z_{d}$ and $Z_{k}$, calculated from (23) and (24) respectively, it is possible to determine a minimum value of the partial safety factor $\gamma_{v \text {,min }}$ for which a randomly implemented headed stud shear resistance will not be underestimated. Such factor can be computed as a ratio:

$$
\begin{aligned}
& \gamma_{v, \min }=\frac{Z_{k}}{Z_{d}}=\exp \left[(3,04-1,645) v_{Z}\right]= \\
& \exp \left(1,395 v_{Z}\right)
\end{aligned}
$$

Let us note that this value depends on the value of a coefficient of variation $v_{Z}$. Simple comparison of results obtained from (24) with a constant value $\gamma_{v}=1,25$ recommended in the standard [4] is given in Fig. 2. As one can see, in case when the variability $v_{Z}$ is large enough (i.e. for $v_{Z}>0,17$ ) a constant value $\gamma_{v}=1,25$ turns out to be insufficient to ensure the required safety level.

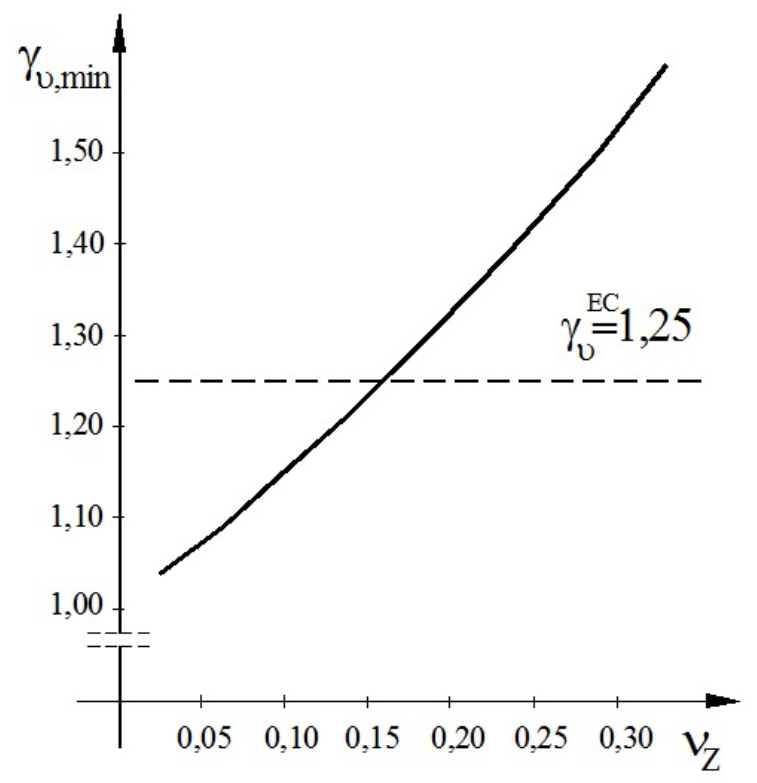

Fig. 2. The minimum values of a partial safety factor $\gamma_{v}$ ensuring the required safety level relating to the random headed stud shear resistance. 


\section{Recommended procedure for determining the design value of a headed stud shear resistance}

In the approach proposed by the authors in this paper the sought design value of a random headed stud shear resistance should be calculated directly from (23). A mean value $\mu_{Z}=E(Z)$ used in such formula is then determined on the basis of (8) while a coefficient of variation $v_{Z}$ - according to (17). To do this, a variation $\sigma_{Z}^{2}=\operatorname{var}(Z)$ has to be specified earlier, as shown in (9), giving in the next step a representative value of the standard deviation $\sigma_{\ln Z}$, based on (15). Alternatively, in (23) the value $\sigma_{\ln Z}^{2}$ can be effectivelly eliminated by the substitution:

$$
\sigma_{\ln Z}^{2}=\ln \left(v_{Z}^{2}+1\right)
$$

The procedure mentioned above is useful in practical application due to the assumption that the boundary $p d f$ functions, both $f_{X}(z)$ and $f_{Y}(z)$, taking into account in (7) to establish the joint $p d f$ function $f_{Z}(z)$, are characterized by the log-normal probability distribution.

\section{Calculation example}

A computational procedure discussed in the previous chapter is illustrated here by an exemplary evaluation of the design value of a random headed stud shear resistance. Let the considered stud has the diameter $d=16 \mathrm{~mm}$ and the length measured after welding $h_{s c}=70 \mathrm{~mm}$. It is assumed that this stub was made of steel for which $f_{u}=400 M P a$. As far as the parameters of the surrounding concrete are concerned these are as follows: $f_{c k}=20 \mathrm{MPa}$ and $E_{c m}=30,5 \mathrm{GPa}$ (being typical for the concrete $\mathrm{C} 20 / 25)$. Such data, after applying the conventional standard formula (1), leads to the evaluation that $P_{R, d}^{E C}=46 \mathrm{kN}$.

Using the new probabilistic approach, however, reveals the relationship between the sought design value of a random headed stud shear resistance $P_{R, d}=Z_{d}$ and the coefficient of variation $v_{Z}$ (in accordance with (23)). This, after taking into account (7) and (9), transformes into appropriate dependences: on the degree of variability $v_{X}=v_{S}$, relating to the strength of steel the considered stud was made of, as well as on the degree of variability $v_{Y}=v_{c}$, corresponding to the surrounding concrete parameters. The sought design values of a random headed stud shear resistance $P_{R, d}=P_{R, d}\left(v_{c}\right)$, obtained in the example for subsequent values of the variability $v_{c}$, with an assumption that the variability of steel strength is constant and set at the level $v_{S}=0,10$, are shown in detail in Fig. 3.

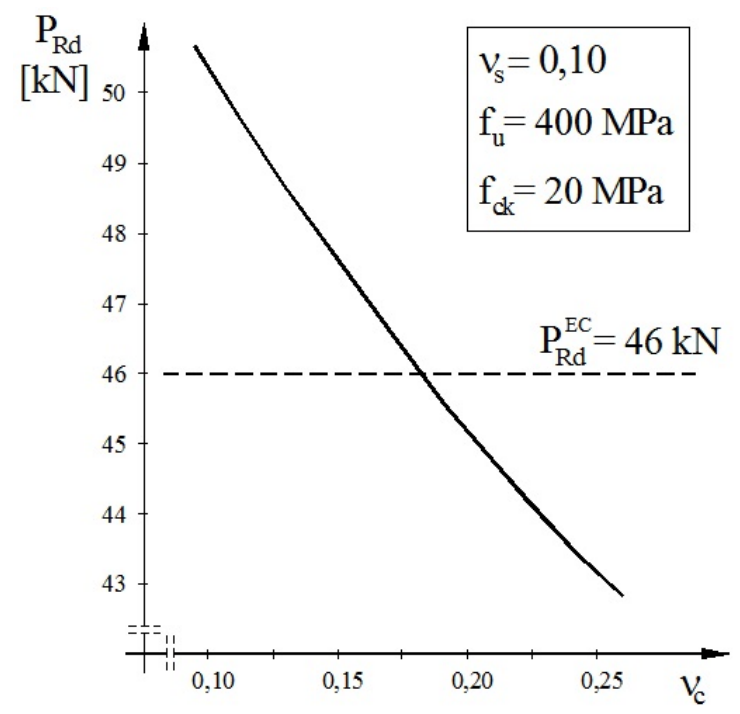

Fig. 3. The design values of a random headed stud shear resistance obtained for the data considered in the example.

\section{Concluding remarks}

The detailed analysis performed by the authors in this paper discloses an important dependence between the design value of a random headed stud shear resistance $P_{R, d}$, established by calculations, and the known a priori values of the coefficients of variation including: $v_{S}$ - relating to the resistance of steel the considered stud is made of, and $v_{c}$ associated with the strength of surrounding concrete, respectively. This type of impact cannot be visualized using only a simplified approach to determine the sought design value, based on the recommendations given in the standard EN 1994-1-1 [4]. Hence, a conclusion may be formulated that the numerical results obtained due to the application of formula (1), without any additional research, can be interpreted at most as the fairly rough estimates of the analoguous values computed in more 
complex way. Such new values seem to be more accurate because these are identified as the relevant quantile of the appropriate probability distribution describing the random nature of a headed stud shear resistance. The quantile of this type is usually determined for the failure probability set at the level $p_{f, u l t} \approx 1,18 \cdot 10^{-3}$, which correspond to the specification that $\beta_{R, r e q}=3,04$.

To unambiguously determine a headed stud shear resistance for a single random implementation a minimum value of two random variables, $P_{R, s}$ and $P_{R, c}$ respectively, should be identified. It is essential that the design value specified for such random minimum is not quantitatively equivalent to the deterministic minimum of the design values $P_{R, s, d}$ and $P_{R, c, d}$, calculated separately, as it is incorrectly recommended in the standard [4]. This means that:

$$
\begin{aligned}
& P_{R, d}=[\min (X, Y)]_{d}=\left[\min \left(P_{R, s}, P_{R, c}\right)\right]_{d} \neq \\
& \neq \min \left(P_{R, s, d}, P_{R, c, d}\right)
\end{aligned}
$$

The results of a numerical example, presented in this paper, allow to conclude that for a fixed value of the variability $v_{S}$, set at the level $v_{s}=0,10$, and with a sufficiently high homogeneity of surrounding concrete (which is equivalent to the specification that the level of the variability $v_{c}$, relating to its strength, is low enough), the design value of a random headed stud shear resistance, determined by the use of fully probabilistic calculations, is higher than the analoguous design value resulting from a simple deterministic comparison of the values
$P_{R, s, d}$ and $P_{R, c, d}$. However, if the concrete surrounding the considered headed stud is identified to be heterogeneous (for instance for the data used in the presented example the variability of its strength in such case should meet the condition that $\left.v_{c}>0,17\right)$ the design value of a random headed stud shear resistance, specified by conventional standard methods, turns out to be clearly overestimated.

\section{References}

[1] Shariati A, Ramli SNH, Suhatril M, Shariati M. Various types of shear connectors in composite structures. A review. International Journal of Physical Sciences 2012;9:7(22):2876-2890.

[2] Olgaard J, Slutter R, Fisher J. Shear strength of stud connectors in lightweight and normalweight concrete. AISC Engineering Journal 1971;8:55-64.

[3] Lam D, Ellobody E. Behavior of headed stud shear connectors in composite beam. Journal of Structural Engineering 2005;131:96.

[4] EN 1994-1-1. Eurocode 4: Design of composite steel concrete structures. Part 1-1: General rules and rules for buildings.

[5] Couchman G. Minimum degree of shear connection rules for UK construction to Eurocode 4. The Steel Construction Institute. SCI P405. Ascot; 2015.

[6] Domański T, Maślak M. Miarodajna obliczeniowa nośność sworznia $\mathrm{w}$ belkach zespolonych, Obiekty Inżynierskie 2009; 2; 2327.

[7] Benjamin JR, Cornell CA. Probability, statistics, and decision for civil engineers. Dover Publications Inc., Mineola, New York; 2014.

[8] EN 1990: Eurocode - Basis of structural design. 